\title{
Response to Cognitive outcome following glioma surgery
}

\author{
Jannick Brennum $^{1,2}$ (1) $\cdot$ Christina M. Engelmann ${ }^{1} \cdot$ Johanne Asperud Thomsen $^{1} \cdot$ Jane Skjøth-Rasmussen $^{1}$ \\ Received: 31 July 2018 / Accepted: 5 August 2018 / Published online: 8 August 2018 \\ (C) Springer-Verlag GmbH Austria, part of Springer Nature 2018
}

We thank Pietro Ivo D'Urso for his appreciation of our study [1] expressed in the above letter to the Editor [2]. We are in complete agreement with Pietro Ivo D'Urso, that the degrees of neurological and cognitive deficits in glioma patients are likely to be underestimated in most publications. We appreciate the questions raised that allow us to clarify our findings and in one case to correct a typo.

We utilized dependency matrices and multiple regression analysis to evaluate factors we a priori found likely to influence the risk of deficits among these tumor grade and adjuvant therapy administered during the post-surgical follow-up period of 1 year. However, none of these factors had any statistically significant influence on the frequency or degree of deficits as stated in the final paragraph of the result section. We did not explore this further. In the discussion, we infer that the reason we did not find any statistically significant influence of the factors tested is likely related to the relative small sample size $(n=92)$

We thank Pietro Ivo D'Urso for identifying a typo in Table 3 for which we apologize. The table should have stated that the procedure was terminated in:

- $55 \%$ because a functional border was reached

- $41 \%$ because complete resection was expected

- $2 \%$ because of patient fatigue

- $2 \%$ due to pain/anxiety

As we discuss in the article, it can be very difficult with certainty to differentiate between affection of eloquent func-

This article is part of the Topical Collection on Tumor - Glioma

Jannick Brennum

jannick.brennum@regionh.dk

1 Department of Neurosurgery, Rigshospitalet, University of Copenhagen, Copenhagen, Denmark

2 Rigshospitalet, University of Copenhagen, Blegdamsvej 9 , DK2100 Copenhagen, Denmark tional pathways and the influence of fatigue, pain, and anxiety. Most of our patients are awake during the final stages of surgery and we regularly experience that the very symptoms that led to the termination of tumor resection revert already by the completion of the procedure. We have wondered whether in these cases the change in brain temperature during the procedure, change in pressure alike to the syndrome of the trephine, and brain shift may be factors influencing the intraoperative performance of the patient.

Regarding the methodology in our classification of deficits and the grading of these deficits, two of us read all the notes in the electronic hospital records that covered all departments involved in the treatment of the patients. We used the definitions given in the methods section of the article. If we rated a deficit different, we chose the most severe grading in order not to underestimate the degree of the deficit. Even so, it is likely that if we had interviewed the patients and their relatives in a structured prospective fashion at each of the follow-up time points, we would have discovered an even higher frequency of deficits.

We hope our study will inspire future studies to address not just major deficits but also minor deficits and patient and relatives' perceived quality of life which will enable us as health care professionals to reach balanced decisions regarding treatment together with our patients.

\section{Compliance with ethical standards}

Conflict of interest The authors declare that they have no conflict of interest.

\section{References}

1. Brennum J, Engelmann CM, Thomson JA, Skjøth-Rasmussen J (2018) Glioma surgery with intraoperative mapping — balancing the onco-functional choice. Acta Neurochir 160:1043-1050

2. D'urso PI (2018) Cognitive outcome following glioma surgery. Acta Neurochir ?? 